\title{
Akt/ERK Inhibitor ONC201
}

National Cancer Institute

\section{Source}

National Cancer Institute. Akt/ERK Inhibitor ONC201. NCI Thesaurus. Code C113792.

A water soluble, orally bioavailable inhibitor of the serine/threonine protein kinase Akt (protein kinase B) and extracellular signal-regulated kinase (ERK), with potential antineoplastic activity. Upon administration, Akt/ERK inhibitor ONC201 binds to and inhibits the activity of Akt and ERK, which may result in inhibition of the phosphatidylinositol 3-kinase (PI3K)/Akt signal transduction pathway as well as the mitogen-activated protein kinase (MAPK)/ERK-mediated pathway. This may lead to the induction of tumor cell apoptosis mediated by tumor necrosis factor (TNF)-related apoptosis-inducing ligand (TRAIL)/T RAIL death receptor type 5 (DR5) signaling in AKT/ERK-overexpressing tumor cells. The PI3K/Akt signaling pathway and MAPK/ERK pathway are upregulated in a variety of tumor cell types and play a key role in tumor cell proliferation, differentiation and survival by inhibiting apoptosis. In addition, ONC201 is able to cross the blood-brain barrier. 